\title{
Principles of Mobile Learning
}

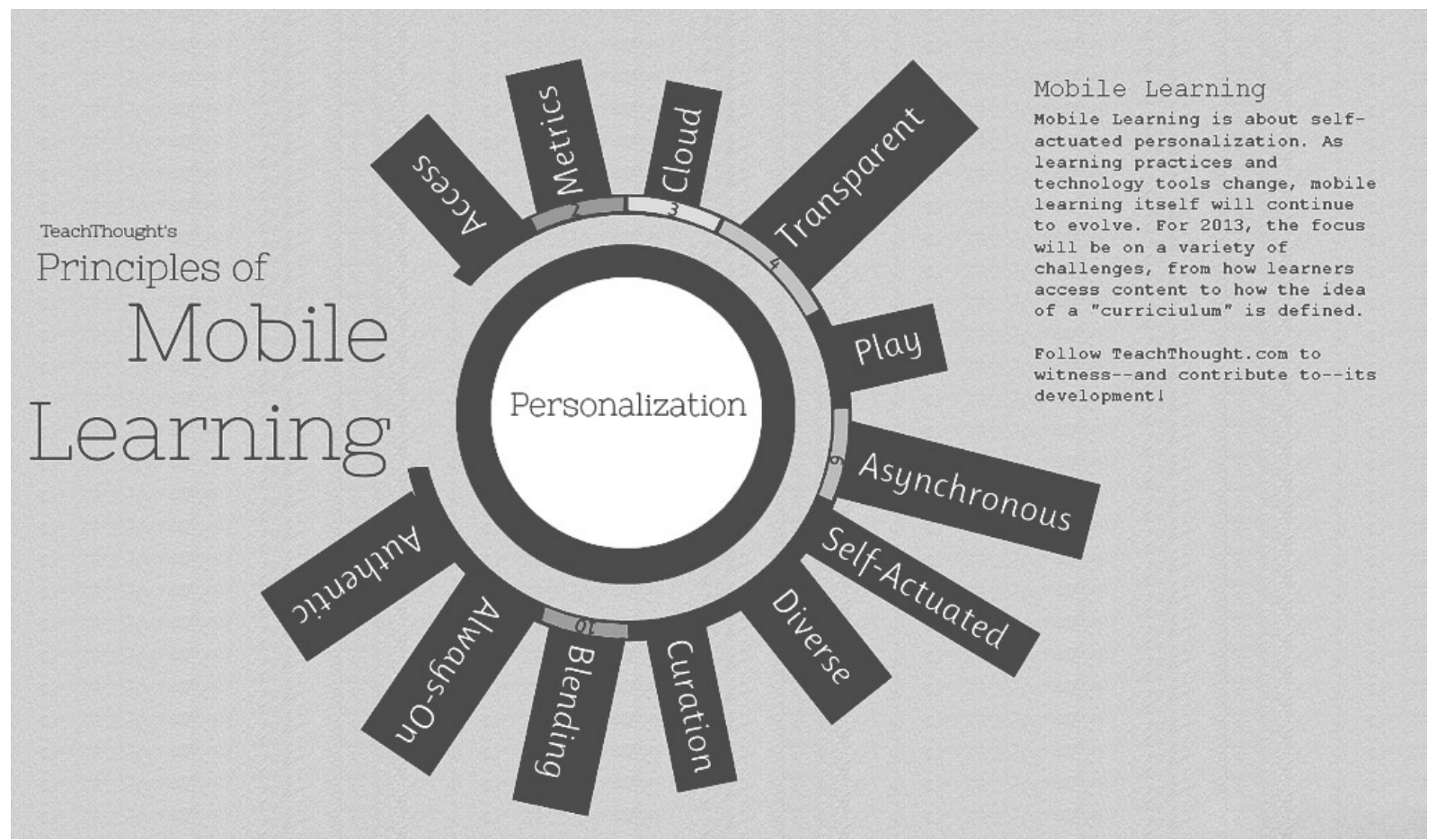

Mobile Learning is about self-actuated personalization.

As learning practices and technology tools change, mobile learning itself will continue to evolve. For 2016, the focus is on a variety of challenges, from how learners access content to how the idea of a "curriculum" is defined. Technology like tablets PCs, apps, and access to broadband internet are lubricating the shift to mobile learning, but a truly immersive mobile learning environment goes beyond the tools for learning to the lives and communities valued by each individual learner.

It is only within these communities that the native context of each learner can be fully understood. Here, in these communities that are both local and digital, a "need to know" is born, knowledge accrues incrementally, progress resonates naturally, and a full picture of each learner as a human being fully emerges.

\section{Access}

A mobile learning environment is about access to content, peers, experts, portfolio artifacts, credible sources, and previous thinking on relevant topics. It can be actuated via a smartphone or iPad, laptop or in-person, but access is constant-which in turn shifts a unique burden to learn on the shoulders of the student.

\section{Metrics}

As mobile learning is a blend of the digital and physical, diverse metrics (i.e., measures) of understanding and "performance of knowledge" will be available.

\section{Cloud}

The cloud is the enabler of "smart" mobility. With access to the cloud, all data sources and project materials are constantly available, allowing for previously inaccessible levels and styles of revision and collaboration.

\section{Transparent}

Transparency is the natural byproduct of connectivity, mobility, and collaboration. As planning, thinking, 
performance, and reflection are both mobile and digital, they gain an immediate audience with both local and global communities through social media platforms from twitter to facebook, Edmodo to Instagram.

5. Play

Play is one of the primary characteristics of authentic, progressive learning, both a cause and effect of an engaged mind. In a mobile learning environment, learners are encountering a dynamic and often unplanned set of data, domains, and collaborators, changing the tone of learning from academic and compliant to personal and playful.

\section{Asynchronous}

Among the most powerful principles of mobile learning is asynchronous access. This unbolts an educational environment from a school floor and allows it to move anywhere, anytime in pursuit of truly entrepreneurial learning. It also enables a learning experience that is increasingly personalized: just in time, just enough, just for me.

\section{Self-Actuated}

With asynchronous access to content, peers, and experts comes the potential for self-actuation. Here, learners plan topic, sequence, audience, and application via facilitation of teachers who now act as experts of resource and assessment.

\section{Diverse}

With mobility comes diversity. As learning environments change constantly, that fluidity becomes a norm that provides a stream of new ideas, unexpected challenges, and constant opportunities for revision and application of thinking. Audiences are diverse, as are the environments data is being gleaned from and deliv- ered.

\section{Curation}

Apps and mobile devices can not only support curation, but can do so better than even the most caffeinelaced teacher might hope to. By design, these technologies adapt to learners, store files, publish thinking, and connect learners, making curation a matter of process rather than ability.

\section{Blending}

A mobile learning environment will always represent a blending of sorts-physical movement, personal communication, and digital interaction.

\section{Always-On}

Always-on learning is self-actuated, spontaneous, iterative, and recursive. There is a persistent need for information access, cognitive reflection, and interdependent function through mobile devices. It is also embedded in communities capable of intimate and natural interaction with students.

12. Authentic

All of the previous 11 principles yield an authenticity to learning that is impossible to reproduce in a classroom. They also ultimately converge to enable experiences that are truly personalized.

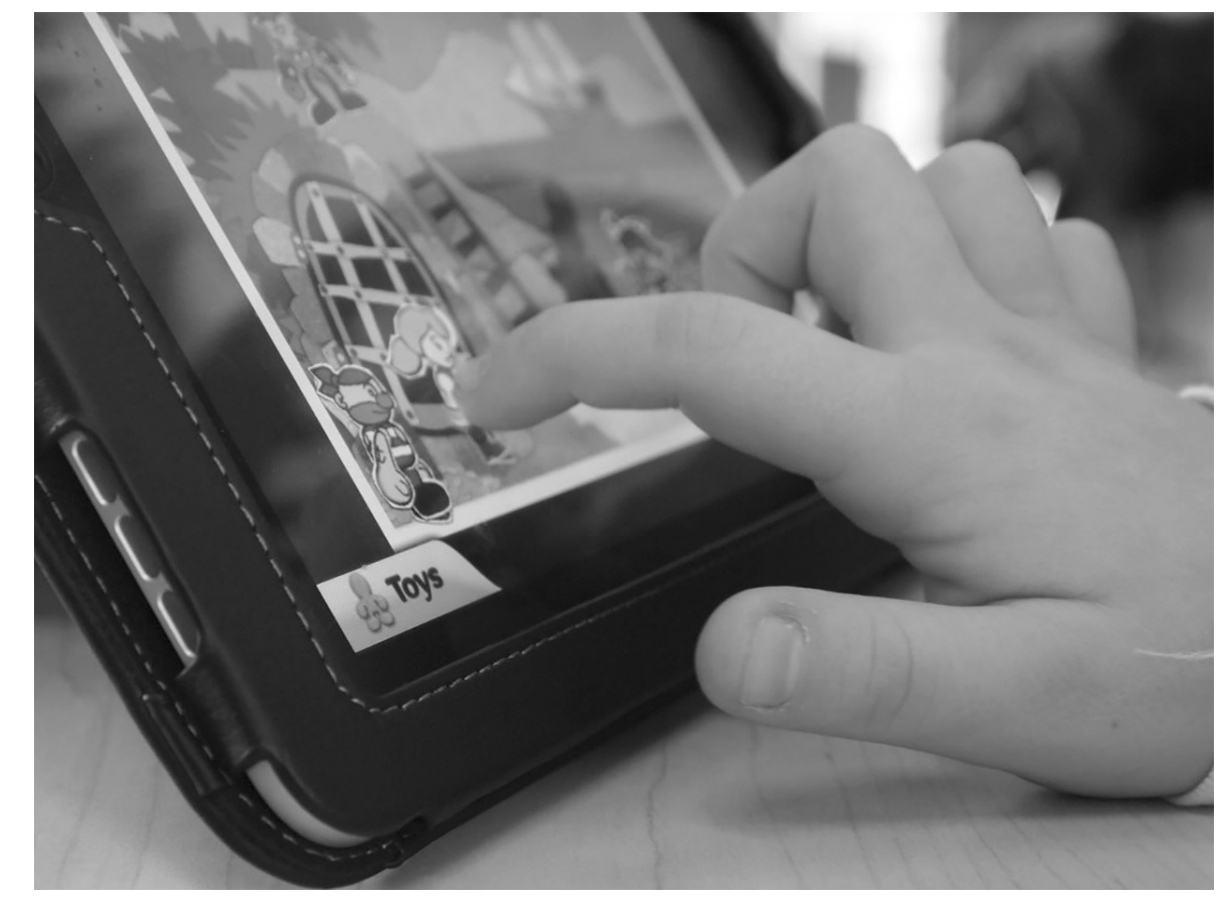

